\title{
铜催化的 5-氯-8-氨基喹啉导向的邻位磺酰化
}

\author{
王向阳门, $a$ 高君青门, $a$ 徐学涛*,a 方 萍 $*, b \quad$ 梅天胜 $^{b}$ \\ $\left({ }^{a}\right.$ 五邑大学生物科技与大健康学院 广东江门 529020) \\ $\left({ }^{b}\right.$ 中国科学院上海有机化学研究所 金属有机化学国家重点实验室 分子合成卓越中心 上海 200032)
}

\begin{abstract}
摘要 砜是天然产物和活性分子中常见的结构, 也是合成反应的重要中间体. 利用 5-氯-8-氨基喹啉(AQ')作为双齿导向 基团, 以各种芳基亚磺酸钠为磺酰化试剂, 通过铜催化实现了邻位 $\mathrm{C}\left(\mathrm{sp}^{2}\right)-\mathrm{H}$ 直接磺酰化. 该反应具有较高的官能团兼 容性和广泛的底物范围, 适用于具有双取代基和稠环的底物. 另外, AQ'作为双齿导向基团易于脱去, 为合成砜类化合 物提供了一种新型的方法. 更重要的是该反应放大至克级规模依然具有良好收率.
\end{abstract}

关键词 铜催化; 碳氢键官能团化; 磺酰化

\section{Copper-Catalyzed ortho-Sulfonylation with 5-Chloro-8- aminoquinoline Group-Directed}

\author{
Wang, Xiangyang ${ }^{\dagger, a} \quad$ Gao, Junqing ${ }^{\dagger, a} \quad \mathrm{Xu}$ Xuetao $^{*, a} \quad$ Fang, Ping $^{*, b} \quad$ Mei, Tiansheng ${ }^{b}$ \\ ( ${ }^{a}$ School of Biotechnology and Health Sciences, Wuyi University, Jiangmen, Guangdong 529020) \\ $\left({ }^{b}\right.$ Center for Excellence in Molecular Synthesis, State Key Laboratory of Organometallic Chemistry, Shanghai Institute of \\ Organic Chemistry, Chinese Academy of Sciences, Shanghai 200032)
}

\begin{abstract}
Sulfone is a common structure in natural products and active molecules, and also an important intermediate in organic synthesis. Sulfonylation is one of the most basic and important reactions in organic synthesis. The direct sulfonylation of $\mathrm{C}\left(\mathrm{sp}^{2}\right)-\mathrm{H}$ bond has been successfully realized by copper catalysis using 5-chloro-8-aminoquinoline (AQ') as a bidentate guiding group and various substituted sodium arylsulfites as sulfonylation reagent. This reaction has high functional group tolerance and a wide scope of substrates, including substrates with double substituents or fused rings. AQ' bidentate guiding group can be removed easily, which provides a new method for the synthesis of sulfone compounds. The reaction could be scaled up to the gram scale with a good yield.

Keywords copper catalysis; carbon hydrogen functionalization; sulfonylation
\end{abstract}

砜是有机分子中一种常见的结构, 广泛存在于各种 天然产物、活性分子和药物分子中 ${ }^{[1]}$, 如 Dapsone ${ }^{[2]}$, Casodex $^{[3]}$, Pyroxasulfone ${ }^{[4]}$, Mesotrione ${ }^{[5]}$ (图 1). 此外, 砜也是有机合成的重要中间体 ${ }^{[6]}$, 如兰堡 - 巴克伦 (Ramberg-Backlund) 反应 ${ }^{[7]}$ 和朱利亚烯烃(Julia olefination)反应 ${ }^{[8]}$. 因此, 砜的合成引起了化学家们的极大关 注 ${ }^{[9]}$. 然而, 传统的方法存在一些缺陷, 比如区域选择 性差、官能团兼容性差以及底物需要预官能团化等 ${ }^{[10]}$. 近二十年来, 过渡金属催化与导向基相结合的 $\mathrm{C}-\mathrm{H}$ 键
官能团化策略作为一种高效以及高区域选择性的方法 被化学家们广泛研究 ${ }^{[11]}$, 该策略也被用于 $\mathrm{C}-\mathrm{S}$ 键的构 建 ${ }^{[12]}$.

2009 年, Dong 课题组 ${ }^{[13]}$ 首次报道了 Pd 催化 2-芳基 吡啶与磺酰氯作为亲电试剂的 $\mathrm{C}\left(\mathrm{sp}^{2}\right)-\mathrm{H}$ 磺酰化反应. 随后，多种过渡金属(如 $\mathrm{Cu} 、 \mathrm{Pd} 、 \mathrm{Ru} 、 \mathrm{Ni} 、 \mathrm{Rh}$ 和 $\mathrm{Co}$ 等) 催化剂, 各类导向基团以及各种磺酰化试剂均被用于砜 的合成研究 ${ }^{[14]}$. 目前能够实现邻位磺酰化反应的例子 不多 ${ }^{[15]}$, 其中, 有些反应需要用到化学计量的催化

\footnotetext{
* Corresponding authors. E-mail: wyuchemxxt@126.com; pfang@sioc.ac.cn Received May 9, 2020; revised July 28, 2020; published online August 5, 2020.

Project supported by the National Natural Science Foundation of China (Nos. 21772222, 21821002) and the Department of Education of Guangdong Province (Nos. 2017KSYS010, 2017KZDXM084, 2019KZDZX2003, 2019KZDXM035).

国家自然科学基金(Nos. 21772222，21821002)和广东省教育厅基金(Nos. 2017KSYS010, 2017KZDXM084, 2019KZDZX2003, 2019KZDXM035)资助项 目.

$\dagger$ 共同第一作者(These authors contributed equally to this work).
} 
<smiles>CO[N+](=O)c1cc(S(=O)(=O)CC(O)(C(=O)Nc2ccc(S(=O)(=O)c3ccc(N)cc3)cc2)C(=O)Nc2ccc(C#N)c(C(F)(F)F)c2)ccc1C(=O)C1C(=O)CCCC1=O</smiles>

图 1 具有砜结构的活性分子和药物

Figure 1 Active molecules and drugs with sulfone structure 剂 ${ }^{[15 a-15 b]}$, 或者需要昂贵的氧化剂 ${ }^{[15 c-15 e]}$, 或者用到不易 操作的磺酰化试剂 ${ }^{[15 f-15 i]}$. 由于铜催化剂价格低廉, 性 质稳定, 在与导向基相结合的 $\mathrm{C}-\mathrm{H}$ 键磺酰化反应中被 广泛应用 ${ }^{[15 a-15 c, 15 f, 16]}$. 但是, 当以 8 -氨基喹啉 $(A Q)$ 作 为导向基团时, 磺酰化反应通常发生在 C-5 位 ${ }^{[16]}$. 因此, 发展利用铜作为催化剂, 使用更容易操作的磺酰化试
剂, 在更温和的氧化体系下实现邻位的磺酰化反应是一 个尚待解决的问题.

\section{1 结果与讨论}

\section{1 反应条件优化}

拟采用 5-氯-8-氨基喹啉作为导向基，利用空气中 的氧气作为氧化剂实现铜催化的 $\mathrm{C}\left(\mathrm{sp}^{2}\right)-\mathrm{H}$ 直接磺酰化. 首先, 以苯甲酰胺衍生物 (1a) 和对甲苯亚磺酸钠 $(\mathbf{2 a})$ 为 模板底物, $N, N$-二甲基甲酰胺(DMF)作为溶剂, $80{ }^{\circ} \mathrm{C}$ 条 件下，尝试了不同的铜催化剂，如 $\mathrm{CuCl} 、 \mathrm{Cu}(\mathrm{OAc})_{2}$ • $\mathrm{H}_{2} \mathrm{O} 、 \mathrm{CuBr}_{2}$ 和 $\mathrm{Cu}(\mathrm{OTf})_{2}$ 均可顺利得到产物(表 1, Entries $1 \sim 4)$; 当使用 $\mathrm{Cu}(\mathrm{OAc})_{2}$ 作为催化剂时, 以 $59 \%$ 的中等 收率得到产物(表 1, Entry 5); 没有铜催化剂, 无法得到 产物(表 1, Entry 6). 值得注意的是，温度降低，产率有 所下降(表 1, Entry 7). 随后对不同的苯甲酸类添加剂进 行了篮选，分别用苯甲酸、3,4-二甲基苯甲酸、2,4,6-三 甲基苯甲酸和 4-叔丁基苯基酸等进行实验，其中苯甲

表 1 反应条件优化 ${ }^{a}$

Table 1 Optimization of the reaction conditions

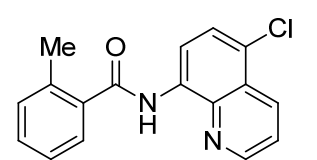

$1 \mathbf{a}$

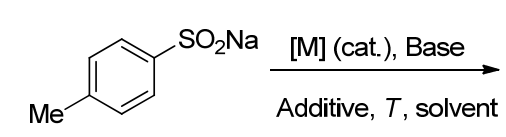

$2 a$<smiles>Cc1cccc(P)c1C(=O)Nc1ccc(Cl)c2cccnc12</smiles>

$3 a$

\begin{tabular}{|c|c|c|c|c|c|}
\hline Entry & {$[\mathrm{M}]$} & Additive & Base & Solvent & Yield $^{b} / \%$ \\
\hline $1^{c}$ & $\mathrm{CuCl}$ & $\mathrm{PhCOOH}$ & KOPiv & DMF & 38 \\
\hline $2^{c}$ & $\mathrm{Cu}(\mathrm{OAc})_{2} \bullet \mathrm{H}_{2} \mathrm{O}$ & $\mathrm{PhCOOH}$ & KOPiv & $\mathrm{DMF}$ & 46 \\
\hline $3^{c}$ & $\mathrm{CuBr}_{2}$ & $\mathrm{PhCOOH}$ & KOPiv & $\mathrm{DMF}$ & 10 \\
\hline $4^{c}$ & $\mathrm{Cu}(\mathrm{OTf})_{2}$ & $\mathrm{PhCOOH}$ & KOPiv & $\mathrm{DMF}$ & 46 \\
\hline $5^{c}$ & $\mathrm{Cu}(\mathrm{OAc})_{2}$ & $\mathrm{PhCOOH}$ & KOPiv & DMF & 59 \\
\hline $6^{c}$ & - & $\mathrm{PhCOOH}$ & KOPiv & DMF & Trace \\
\hline $7^{c d}$ & $\mathrm{Cu}(\mathrm{OAc})_{2}$ & $\mathrm{PhCOOH}$ & KOPiv & $\mathrm{DMF}$ & 45 \\
\hline $8^{e}$ & $\mathrm{Cu}(\mathrm{OAc})_{2}$ & 3,4-Dimethylbenzoic acid & KOPiv & DMF & 66 \\
\hline $9^{e}$ & $\mathrm{Cu}(\mathrm{OAc})_{2}$ & 2,4,6-Trimethylbenzoic acid & KOPiv & DMF & 57 \\
\hline $10^{e}$ & $\mathrm{Cu}(\mathrm{OAc})_{2}$ & 4-tert-Butylbenzoic acid & KOPiv & DMF & 58 \\
\hline $11^{e}$ & $\mathrm{Cu}(\mathrm{OAc})_{2}$ & $\mathrm{PhCOOH}$ & KOPiv & $\mathrm{DMF}$ & 72 \\
\hline $12^{f}$ & $\mathrm{Cu}(\mathrm{OAc})_{2}$ & $\mathrm{PhCOOH}$ & KOPiv & $\mathrm{DMF}$ & 62 \\
\hline 13 & $\mathrm{Cu}(\mathrm{OAc})_{2}$ & $\mathrm{PhCOOH}$ & KOPiv & DMF & 71 \\
\hline 14 & $\mathrm{Cu}(\mathrm{OAc})_{2}$ & $\mathrm{PhCOOH}$ & - & DMF & 13 \\
\hline 15 & $\mathrm{Cu}(\mathrm{OAc})_{2}$ & $\mathrm{PhCOOH}$ & KOAc & DMF & 60 \\
\hline 16 & $\mathrm{Cu}(\mathrm{OAc})_{2}$ & $\mathrm{PhCOOH}$ & $\mathrm{NaOPiv} \cdot \mathrm{H}_{2} \mathrm{O}$ & DMF & 61 \\
\hline 17 & $\mathrm{Cu}(\mathrm{OAc})_{2}$ & $\mathrm{PhCOOH}$ & $\mathrm{K}_{2} \mathrm{CO}_{3}$ & DMF & 36 \\
\hline 18 & $\mathrm{Cu}(\mathrm{OAc})_{2}$ & $\mathrm{PhCOOH}$ & KOPiv & DMSO & 66 \\
\hline 19 & $\mathrm{Cu}(\mathrm{OAc})_{2}$ & $\mathrm{PhCOOH}$ & KOPiv & DCE & 17 \\
\hline 20 & $\mathrm{Cu}(\mathrm{OAc})_{2}$ & $\mathrm{PhCOOH}$ & KOPiv & $\mathrm{CH}_{3} \mathrm{CN}$ & 46 \\
\hline 21 & $\mathrm{Cu}(\mathrm{OAc})_{2}$ & $\mathrm{PhCOOH}$ & KOPiv & 1,4-Dioxane & 17 \\
\hline 22 & $\mathrm{Cu}(\mathrm{OAc})_{2}(10 \mathrm{~mol} \%)$ & PhCOOH (20 mol\%) & KOPiv & DMF & 58 \\
\hline 23 & $\mathrm{Cu}(\mathrm{OAc})_{2}(20 \mathrm{~mol} \%)$ & PhCOOH (30 mol\%) & KOPiv & DMF & $81(79)^{g}$ \\
\hline 24 & $\mathrm{Cu}(\mathrm{OAc})_{2}(20 \mathrm{~mol} \%)$ & $\mathrm{PhCOOH} \mathrm{(40} \mathrm{mol \% )}$ & KOPiv & DMF & 73 \\
\hline
\end{tabular}

${ }^{a}$ 1a $(0.2 \mathrm{mmol}), \mathbf{2 a}\left(2\right.$ equiv.), [M] catalyst $(20 \mathrm{~mol} \%)$, additive $(20 \mathrm{~mol} \%)$, base (2 equiv.), $1 \mathrm{~mL}$ of solvent, $80{ }^{\circ} \mathrm{C}, 8 \mathrm{~h} ;{ }^{b} \mathrm{Yield} \mathrm{determined} \mathrm{by}^{1} \mathrm{H} \mathrm{NMR} \mathrm{with} \mathrm{CH}{ }_{2} \mathrm{Br}_{2}$ as internal standard; ${ }^{c} \mathbf{2 a}$ (4 equiv.), base (1 equiv.), $24 \mathrm{~h} ;{ }^{d} 70{ }^{\circ} \mathrm{C} ;{ }^{e} 12 \mathrm{~h} ;{ }^{f} 6 \mathrm{~h} ;{ }^{g}$ Isolated yield in parentheses. 
酸取得了最好的收率 $72 \%$ (表 1, Entries 8～11). 缩短反 应时间时，产率有所增加，但当反应时间过短时，产率 反而会下降, 故选出最佳反应时间 $8 \mathrm{~h}$ (表 1, Entries 11 13). 反应体系中没有碱存在时, 产率仅为 $13 \%$, 推测碱 的加入有助于铜的配位, 故而对 $\mathrm{KOAc} 、 \mathrm{NaOPiv} \cdot \mathrm{H}_{2} \mathrm{O}$ 、 $\mathrm{K}_{2} \mathrm{CO}_{3}$ 和 KOPiv 等不同的碱进行耖选, 除 KOPiv 外, 均 未取得较好的结果(表 1, Entries 13～17). 接下来考察了 溶剂对反应的影响, 发现 $N, N$-二甲基甲酰胺作为溶剂时 效果最优(表 1, Entries 13、18～21). 最后对铜催化剂和 苯甲酸添加剂的投料比例进行考察，当使用 $20 \mathrm{~mol} \%$ 铜 催化剂和 $30 \mathrm{~mol} \%$ 苯甲酸时, 以 $79 \%$ 的分离收率得到产 物(表 1, Entries 22 24). 因此选用 $20 \mathrm{~mol} \%$ 铜催化剂、 $30 \mathrm{~mol} \%$ 苯甲酸添加剂、KOPiv 作为碱、 DMF 作溶剂, $80{ }^{\circ} \mathrm{C}$ 条件下反应 $8 \mathrm{~h}$ 作为最优条件.

\section{2 反应底物扩展}

确定最优条件之后, 对反应底物的普适性进行了探 究. 首先考察了苯甲酰胺衍生物(表 2), 各种官能团(烷 基、三氟甲基、苯基、氟、氯以及菜基等)对该反应体 系都能很好地兼容, 以中等至良好的收率得到相应的磺 酰化产物 (3a $\sim 3 \mathbf{j})$. 另外, 双烷基取代(3k)和醚取代(3l) 的苯甲酰胺衍生物也能以良好的收率得到产物. 值得一 提的是稠环类的菜基 $(\mathbf{3 m})$ 也能以中等收率得到相应的 产物.
接下来考察了亚磺酸盐的普适性(表 3). 中性的苯 基亚磺酸钠 $(4 a) 、 F 、 C l 、 B r$ 等卤素 $(4 b \sim 4 d)$ 、吸电子的 三氟甲基 $(4 \mathrm{e})$ 以及供电子烷氧基(4f)和烷基(4g)取代的苯 基亚磺酸钠都能以较高收率得到产物。同时稠环类的荎 基亚磺酸钠(4h)也能以良好收率得到产物.

\section{3 克级规模实验}

为了进一步说明此反应在合成上的实用性, 将反应 放大至克级规模(Scheme 1), 1a (4 mmol, $1.18 \mathrm{~g}$ )在标准 条件下能以 $60 \%$ 的分离收率得到了相应的磺酰化产物 3a $(1.08 \mathrm{~g})$, 显示了该方法具有潜在的实用价值.

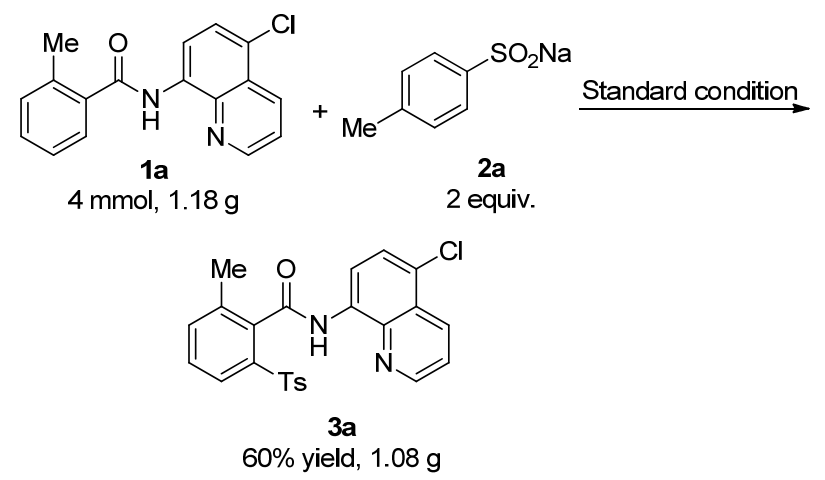

图式 1 克级规模反应

Scheme 1 Gram scale reaction

表 2 苯甲酰胺衍生物的底物范围

Table 2 Substrate scope of benzamide derivative

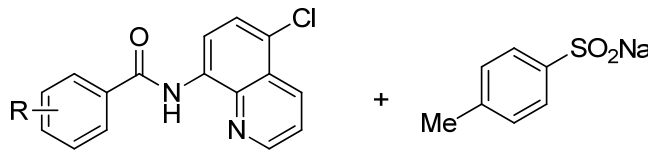

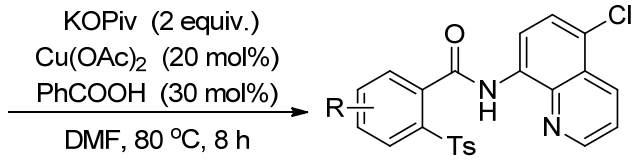

3<smiles>Cc1cccc(F)c1C(=O)Nc1ccc(Cl)c2cccnc12</smiles>

3a, $79 \%$<smiles>O=C(Nc1ccc(Cl)c2cccnc12)c1cc(C(F)(F)F)ccc1F</smiles>

$3 e, 53 \%$<smiles>[3H]c1cc(C(C)(C)C)ccc1C(=O)Nc1ccc(Cl)c2cccnc12</smiles>

3j, $50 \%$<smiles>O=C(Nc1ccc(Cl)c2cccnc12)c1c(F)cccc1C(F)(F)F</smiles>

3b, $75 \%$<smiles>[R]c1ccc(C(=O)Nc2ccc(Cl)c3cccnc23)c([AsH])c1</smiles>

3f: $R=F, 70 \%$ 3g: $\mathrm{R}=\mathrm{Cl}, 51 \%$

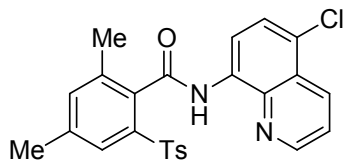

3k, $78 \%$<smiles>O=C(Nc1ccc(Cl)c2cccnc12)c1c(F)cccc1-c1ccccc1</smiles>

3c, $45 \%$<smiles>COc1ccc(C(=O)Nc2ccc(Cl)c3cccnc23)c([15F])c1</smiles>

$3 h, 54 \%$<smiles>O=C(Nc1ccc(Cl)c2cccnc12)c1c(F)ccc2c1OCCO2</smiles>

3I, $71 \%$<smiles>Cc1ccc(F)c(C(=O)Nc2ccc(Cl)c3cccnc23)c1</smiles>

$3 d, 62 \%$<smiles>CCc1ccc(C(=O)Nc2ccc(Cl)c3cccnc23)c(F)c1</smiles>

$3 i, 51 \%$

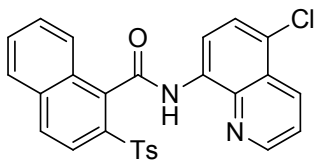

$3 \mathrm{~m}, 46 \%$ 
表 3 芳基亚磺酸钠的底物范围

Table 3 Substrate scope of sodium arylsulfinate
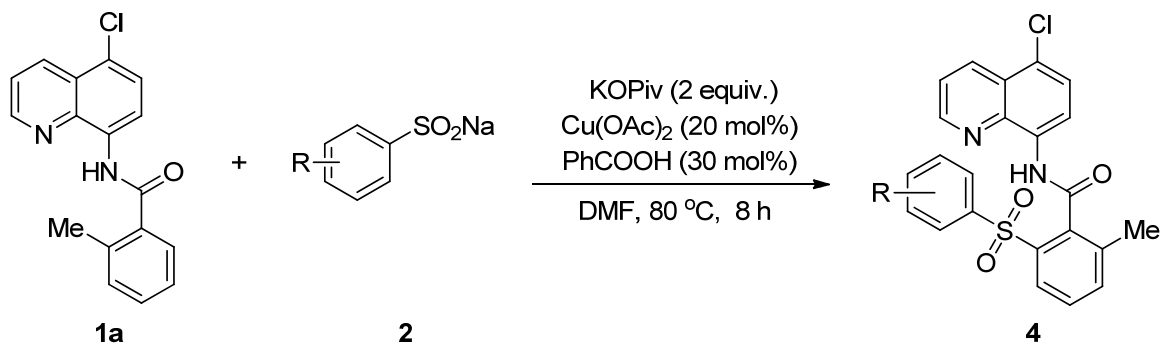<smiles>Cc1cccc(S(=O)(=O)c2ccccc2)c1C(=O)Nc1ccc(Cl)c2cccnc12</smiles>

$4 a, 64 \%$<smiles>Cc1cccc(S(=O)(=O)c2ccc(C(F)(F)F)cc2)c1C(=O)Nc1ccc(Cl)c2cccnc12</smiles>

4e, $78 \%$<smiles>Cc1cccc(S(=O)(=O)c2ccc(F)cc2)c1C(=O)Nc1ccc(Cl)c2cccnc12</smiles>

4b, $61 \%$<smiles>COc1ccc(S(=O)(=O)c2cccc(C)c2C(=O)Nc2ccc(Cl)c3cccnc23)cc1</smiles>

4f, $66 \%$<smiles>Cc1cccc(S(=O)(=O)c2ccc(Cl)cc2)c1C(=O)Nc1ccc(Cl)c2cccnc12</smiles>

$4 c, 64 \%$<smiles>Cc1cccc(S(=O)(=O)c2ccc(C(C)(C)C)cc2)c1C(=O)Nc1ccc(Cl)c2cccnc12</smiles>

$\mathbf{4 g}, 61 \%$<smiles>Cc1cccc(S(=O)(=O)c2ccc(Br)cc2)c1C(=O)Nc1ccc(Cl)c2cccnc12</smiles>

4d, $62 \%$<smiles>Cc1cccc(S(=O)(=O)c2ccc3ccccc3c2)c1C(=O)Nc1ccc(Cl)c2cccnc12</smiles>

4h, $79 \%$

\section{$1.4 \mathrm{AQ}^{\prime}$ 导向基团脱除实验}

为了得到脱去 8-氨基喹啉引导基团的产物，参考了 Tan 课题组 ${ }^{[15 b]}$ 的相关研究. 首先将苯甲酰胺衍生物 $\mathbf{3 g}$ 仲酰胺甲基化生成中间体 $\mathbf{5}$, 然后 $\mathbf{5}$ 在碱性条件下进行 水解, 成功得到了苯甲酸衍生物 6 (Scheme 2). AQ'作为 双齿导向基团易于脱去，为合成砜类化合物提供了一种 新型的方法.<smiles>O=C(Nc1ccc(Cl)c2cccnc12)c1ccc(Cl)cc1Br</smiles>

$3 g$<smiles>CCON[C@H](O)[C@H](O)OCC</smiles>

$$
\text { 6, } 57 \%
$$

图式 $2 \mathrm{AQ}^{\prime}$ 导向基团的脱除

Scheme 2 Removal of the AQ' directing group

\section{5 反应机理探讨}

为了研究反应的机理, 进行了相关实验. 标准条件 下, 加入四甲基哌啶氮氧化物(TEMPO)或 1,1-二苯乙烯,
反应收率明显下降; $\mathbf{2} \mathbf{a}$ 和 1,1-二苯乙烯在标准条件下的 反应可得到自由基产物 7(通过 HRMS 检测到化合物 7 对应的峰), 这一结果暗示反应可能经历了自由基过程 (Scheme 3).

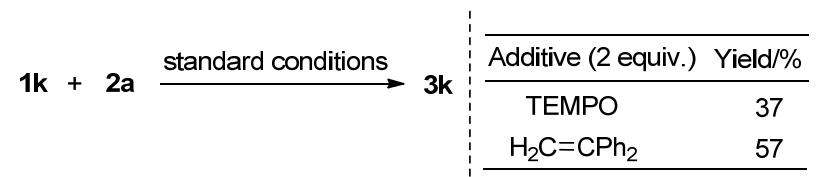

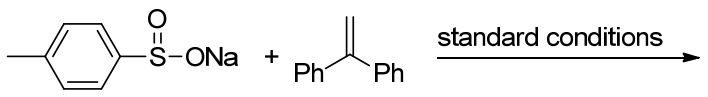

2a<smiles>Cc1ccc(S(=O)(=O)C=C(c2ccccc2)c2ccccc2)cc1</smiles>

图式 3 自由基捕获剂实验

Scheme 3 Radical scavenger reactions

根据以上实验结果，并参考相关的报道 ${ }^{[141,15 \mathrm{c}, 17]}$, 我 们对该反应历程提出了可能的机理(Scheme 4). 苯甲酰 胺 $1 \mathbf{a}$ 与醋酸铜配位, 形成中间体 $\mathbf{A}$, 然后发生碳氢键活 化得到环金属中间体 B. $2 \mathbf{a}$ 在氧气作用下形成磺酰自由 
基, 接下来, 一种可能的途径是: 与中间体 $\mathbf{B}$ 氧化加成 形成中间体 $\mathrm{Cu}(\mathrm{III})$ 物种 $\mathbf{C}$, 随后 $\mathbf{C}$ 还原消除得到磺酰化 产物 3a 和 $\mathrm{Cu}(\mathrm{I})$ 物种; 另一种可能的途径是: 与中间体 B 直接反应生成产物 $\mathbf{3 a}$ 和 $\mathrm{Cu}(\mathrm{I})$ 物种. 最后, $\mathrm{Cu}(\mathrm{I})$ 被 $\mathrm{O}_{2}$ 氧化生成的醋酸铜继续参与到下一个催化循环.

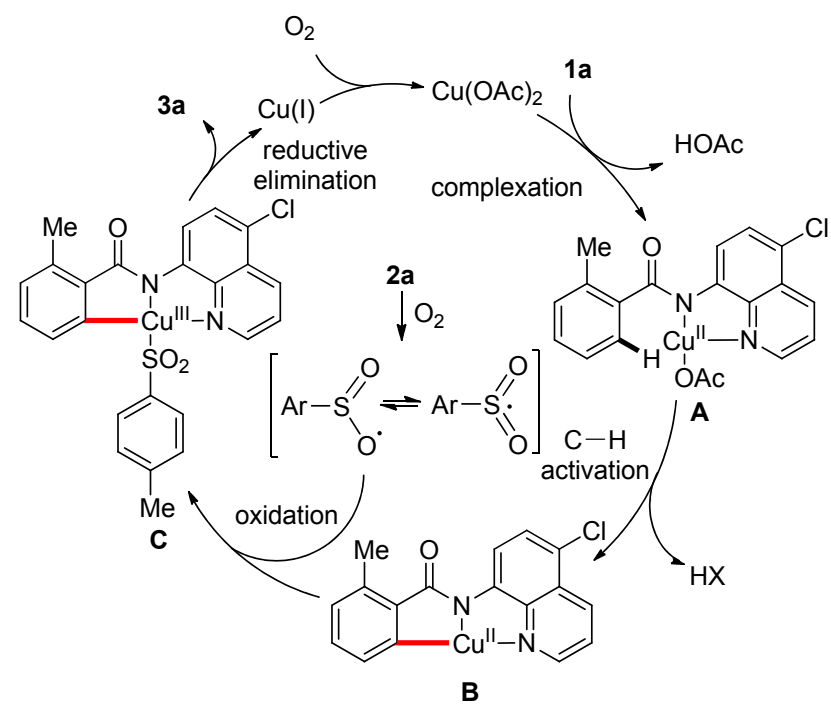

图式 4 可能的反应机理 Scheme 4 Proposed mechanism

\section{2 结论}

以 $\mathrm{AQ}^{\prime}$ 为双齿导向基团, 以空气中的氧气为氧化剂, 以各种取代的苯基亚磺酸钠为磺酰化试剂, 通过铜催化 成功实现了苯甲酰胺类化合物的邻位 $\mathrm{C}\left(\mathrm{sp}^{2}\right)-\mathrm{H}$ 的直接 磺酰化. 此反应条件官能团耐受性强, 底物适用范围广, 同时能兼容双取代和稠环类底物. 此外, 该反应放大至 克级规模依然具有良好收率.

\section{3 实验部分}

\section{1 仪器与试剂}

${ }^{1} \mathrm{H}$ NMR 、 ${ }^{13} \mathrm{C}$ NMR 和 ${ }^{19} \mathrm{~F}$ NMR 在 Varian 400 -MR (400 MHz)或 Agilent 400-MR (400 MHz)型核磁共振仪 上测定, 用 TMS $(\delta 0.00)$ 或 $\mathrm{CDCl}_{3}\left(\delta\right.$ 7.26) 作为 ${ }^{1} \mathrm{H}$ NMR 的内标, $\mathrm{CDCl}_{3}\left(\delta\right.$ 77.00)作为 ${ }^{13} \mathrm{C} \mathrm{NMR}$ 的内标. 红外光 谱(IR)用 Bio-Rad FTS-185 仪器测定. 使用 Agilent Technologies 6224 TOF LC/MS 质谱仪获得了高分辨率 质谱图. 熔点由 WRS-2 微机熔点仪测定. 柱层析使用 300 400 目硅胶. 所有商业试剂均购自 TCI, 阿拉丁, 毕得, Sigma-Aldrich 和 Adamas-beta 等试剂商. 除另有 说明外, 无需进一步纯化即可使用.

\section{2 磺酰化反应条件}

向干燥的反应管中依次加入搅拌子、 DMF $(1 \mathrm{~mL})$ 、 苯甲酰胺衍生物 1 (0.2 mmol)、芳基亚磺酸钠 2 (0.4 $\mathrm{mmol}) 、$ 醋酸铜 $(7.2 \mathrm{mg}, 0.02 \mathrm{mmol}) 、$ 苯甲酸 $(7.4 \mathrm{mg}, 0.03$ $\mathrm{mmol}$ )和特戊酸钾 $(56.0 \mathrm{mg}, 0.4 \mathrm{mmol})$, 将反应体系置于 $80{ }^{\circ} \mathrm{C}$ 油浴中反应 $8 \mathrm{~h}$. 反应结束后冷却至室温, 用乙酸 乙酯 $(5 \mathrm{~mL})$ 稀释后, 再用饱和食盐水 $(20 \mathrm{~mL})$ 洗涤, 合并 有机相并用无水 $\mathrm{Na}_{2} \mathrm{SO}_{4}$ 干燥, 过滤, 减压浓缩, 残余物 通过硅胶柱层析(石油醚/乙酸乙酯, $V: V=3: 1$ )分离得 到目标产物 3 或 4.

$N$-(5-氯-8-喹啉基)-2-甲基-6-甲苯磺酰基苯甲酰胺 (3a): 白色固体，收率 79\%. m.p. 215.8 217.9 ${ }^{\circ} \mathrm{C} ;{ }^{1} \mathrm{H}$ NMR $\left(400 \mathrm{MHz}, \mathrm{CDCl}_{3}\right) \delta$ : $9.87(\mathrm{~s}, 1 \mathrm{H}), 8.90(\mathrm{~d}, J=8.4$ $\mathrm{Hz}, 1 \mathrm{H}), 8.76$ (dd, $J=4.4,1.6 \mathrm{~Hz}, 1 \mathrm{H}), 8.59$ (dd, $J=8.4$, $1.6 \mathrm{~Hz}, 1 \mathrm{H}), 8.01(\mathrm{t}, J=4.4 \mathrm{~Hz}, 1 \mathrm{H}), 7.82(\mathrm{~d}, J=8.0 \mathrm{~Hz}$, $2 \mathrm{H}), 7.69(\mathrm{~d}, J=8.4 \mathrm{~Hz}, 1 \mathrm{H}), 7.63 \sim 7.55(\mathrm{~m}, 1 \mathrm{H}), 7.53 \sim$ 7.46 (m, 2H), 7.18 (d, $J=8.0 \mathrm{~Hz}, 2 \mathrm{H}), 2.44$ (s, 3H), 2.32 $(\mathrm{s}, 3 \mathrm{H}) ;{ }^{13} \mathrm{C}$ NMR $\left(100 \mathrm{MHz}, \mathrm{CDCl}_{3}\right) \delta: 165.6,148.9$, $144.3,139.1,138.9,138.4,137.3,136.2,135.6,133.7$, $133.5,129.8,129.7,128.3,127.4,127.2,126.1,125.1$, 122.5, 117.0, 21.7, 19.5; IR (neat) $v: 3301,2921,1680$, 1524, 1483, 1328, 1310, 1137, 1086, 940, 661, $578 \mathrm{~cm}^{-1}$; HRMS (ESI-TOF) calcd for $\mathrm{C}_{24} \mathrm{H}_{17}-\mathrm{N}_{2} \mathrm{O}_{3} \mathrm{~F}_{3} \mathrm{SCl}[\mathrm{M}+\mathrm{H}]^{+}$ 505.0600 , found 505.0590 .

$N$-(5-氯-8-喹啉基)-2-甲苯磺酰基-6-(三氟甲基)苯甲 酰胺 (3b): 淡黄色固体, 收率 75\%. m.p. 223.7 $225.6{ }^{\circ} \mathrm{C} ;{ }^{1} \mathrm{H}$ NMR $\left(400 \mathrm{MHz}, \mathrm{CDCl}_{3}\right) \delta: 10.06(\mathrm{~s}, 1 \mathrm{H})$, $8.84(\mathrm{~d}, J=8.4 \mathrm{~Hz}, 1 \mathrm{H}), 8.77$ (dd, $J=4.0,1.6 \mathrm{~Hz}, 1 \mathrm{H})$, 8.59 (dd, $J=8.4,1.6 \mathrm{~Hz}, 1 \mathrm{H}), 8.45(\mathrm{~d}, J=8.0 \mathrm{~Hz}, 1 \mathrm{H})$, $7.97(\mathrm{~d}, J=8.0 \mathrm{~Hz}, 1 \mathrm{H}), 7.84(\mathrm{~d}, J=8.4 \mathrm{~Hz}, 2 \mathrm{H}), 7.77(\mathrm{t}$, $J=8.0 \mathrm{~Hz}, 1 \mathrm{H}), 7.70$ (d, $J=8.4 \mathrm{~Hz}, 1 \mathrm{H}), 7.57$ (dd, $J=8.4$, $4.0 \mathrm{~Hz}, 1 \mathrm{H}), 7.22(\mathrm{~d}, J=8.0 \mathrm{~Hz}, 2 \mathrm{H}), 2.33(\mathrm{~s}, 3 \mathrm{H}) ;{ }^{13} \mathrm{C}$ NMR $\left(100 \mathrm{MHz}, \mathrm{CDCl}_{3}\right) \delta: 162.1,149.0,145.1,141.3$, 139.0, 137.4, 134.6 (q, $J=2.0 \mathrm{~Hz}), 133.6,133.5,133.5$, $131.3(\mathrm{q}, J=4.0 \mathrm{~Hz}), 130.5,130.0,129.5(\mathrm{q}, J=31.0 \mathrm{~Hz})$, 128.6, 127.4, 126.1, 125.5, 123.0 (q, $J=274.0 \mathrm{~Hz}$ ), 122.6, 117.1, 21.8; ${ }^{19} \mathrm{~F}$ NMR (376 MHz, $\left.\mathrm{CDCl}_{3}\right) \delta:-58.68$; IR (neat) $v: 3301,2921,1680,1524,1483,1328,1310,1137$, 1086, 940, 661, $578 \mathrm{~cm}^{-1}$; HRMS (ESI-TOF) calcd for $\mathrm{C}_{24} \mathrm{H}_{17} \mathrm{~N}_{2} \mathrm{O}_{3} \mathrm{~F}_{3} \mathrm{SCl}[\mathrm{M}+\mathrm{H}]^{+}$505.0600, found 505.0590 .

$N$-(5-氯-8-喹啉基)-3-甲苯磺酰基-[1,1'-联苯]-2-羧酰 胺(3c): 黄色固体, 收率 $45 \%$. m.p. $199.3 \sim 202.4{ }^{\circ} \mathrm{C} ;{ }^{1} \mathrm{H}$ NMR (400 MHz, $\left.\mathrm{CDC}_{13}\right) \delta: 9.61$ (s, 1H), 8.63 (d, $J=8.0$ $\mathrm{Hz}, 2 \mathrm{H}), 8.46(\mathrm{~d}, J=8.4 \mathrm{~Hz}, 1 \mathrm{H}), 8.25(\mathrm{~d}, J=6.4 \mathrm{~Hz}$, 1H), 7.94 (d, $J=8.0 \mathrm{~Hz}, 2 \mathrm{H}), 7.65$ (s, 2H), 7.56 (d, $J=8.4$ $\mathrm{Hz}, 1 \mathrm{H}), 7.43$ (d, $J=8.0 \mathrm{~Hz}, 3 \mathrm{H}), 7.23(\mathrm{~d}, J=8.0 \mathrm{~Hz}, 2 \mathrm{H})$, $7.18(\mathrm{t}, J=7.2 \mathrm{~Hz}, 2 \mathrm{H}), 7.11(\mathrm{~d}, J=7.2 \mathrm{~Hz}, 1 \mathrm{H}), 2.32$ (s, $3 \mathrm{H}) ;{ }^{13} \mathrm{C}$ NMR $\left(100 \mathrm{MHz}, \mathrm{CDCl}_{3}\right) \delta: 164.8,148.6,144.4$, 
$141.8,139.7,138.9,138.5,138.3,135.5,135.4,133.6$, $133.2,129.9,129.7,128.9,128.6,128.3,128.1,127.2$, $125.8,124.7,122.3,116.6,21.7$; IR (neat) $v: 3347,3183$, 2919, 1660, 1517, 1474, 1319, 1139, 1086, 732, 664, 756 $\mathrm{cm}^{-1}$; HRMS (ESI-TOF) calcd for $\mathrm{C}_{29} \mathrm{H}_{22} \mathrm{~N}_{2} \mathrm{O}_{3} \mathrm{SCl}[\mathrm{M}+$ $\mathrm{H}]^{+}$513.1034, found 513.1030.

$N$-(5-氯-8-喹啉基)-5-甲基-2-甲苯磺酰基苯甲酰胺 (3d): 白色固体, 收率 $62 \%$. m.p. 82.3 84.5 ${ }^{\circ} \mathrm{C}$ (lit. ${ }^{[16]}$ $\left.90{ }^{\circ} \mathrm{C}\right) ;{ }^{1} \mathrm{H}$ NMR $\left(400 \mathrm{MHz}, \mathrm{CDCl}_{3}\right) \delta: 10.01(\mathrm{~s}, 1 \mathrm{H})$, $8.82(\mathrm{~d}, J=8.4 \mathrm{~Hz}, 1 \mathrm{H}), 8.78(\mathrm{dd}, J=4.4,1.6 \mathrm{~Hz}, 1 \mathrm{H})$, $8.58(\mathrm{dd}, J=8.4,1.6 \mathrm{~Hz}, 1 \mathrm{H}), 8.07$ (d, $J=8.4 \mathrm{~Hz}, 1 \mathrm{H})$, $7.87 \sim 7.78(\mathrm{~m}, 2 \mathrm{H}), 7.67(\mathrm{~d}, J=8.4 \mathrm{~Hz}, 1 \mathrm{H}), 7.56(\mathrm{dd}$, $J=8.4,4.0 \mathrm{~Hz}, 1 \mathrm{H}), 7.41(\mathrm{dd}, J=5.6,2.0 \mathrm{~Hz}, 2 \mathrm{H}), 7.19$ (d, $J=8.0 \mathrm{~Hz}, 2 \mathrm{H}), 2.44$ (s, 3H), 2.32 (s, 3H); ${ }^{13} \mathrm{C} \mathrm{NMR}$ $\left(100 \mathrm{MHz}, \mathrm{CDCl}_{3}\right) \delta: 166.0,148.9,144.8,144.3,139.1$, $138.4,136.9,136.2,133.8,133.5,131.0,129.9,129.7$, $129.4,128.3,128.3,127.3,126.1,125.1,122.5,117.1$, 21.7; IR (neat) v: 2920, 1678, 1518, 1476, 1384, 1317, 1152, 1089, 947, 675, 627, $548 \mathrm{~cm}^{-1}$; HRMS (ESI-TOF) calcd for $\mathrm{C}_{24} \mathrm{H}_{20} \mathrm{~N}_{2} \mathrm{O}_{3} \mathrm{SCl}[\mathrm{M}+\mathrm{H}]^{+}$451.0878, found 451.0879 .

$N$-(5-氯-8-喹啉基)-2-甲苯磺酰基-5-(三氟甲基)苯甲 酰胺(3e): 黄色固体, 收率 53\%. m.p. 209.2 212.3 ${ }^{\circ} \mathrm{C}$ (lit. ${ }^{[16]} 213{ }^{\circ} \mathrm{C}$ ); ${ }^{1} \mathrm{H}$ NMR (400 MHz, $\mathrm{CDCl}_{3}$ ) $\delta: 10.12$ (s, $1 \mathrm{H}), 8.86 \sim 8.72(\mathrm{~m}, 2 \mathrm{H}), 8.62 \sim 8.54(\mathrm{~m}, 1 \mathrm{H}), 8.34(\mathrm{~d}, J=$ $8.4 \mathrm{~Hz}, 1 \mathrm{H}), 7.89$ (s, 1H), 7.85 (d, J=8.0 Hz, 2H), 7.68 (d, $J=8.4 \mathrm{~Hz}, 1 \mathrm{H}), 7.58(\mathrm{dd}, J=8.4,4.0 \mathrm{~Hz}, 1 \mathrm{H}), 7.29 \sim 7.18$ (m, 2H), $2.34(\mathrm{~s}, 3 \mathrm{H}) ;{ }^{13} \mathrm{C} \mathrm{NMR}\left(100 \mathrm{MHz}, \mathrm{cdcl}_{3}\right) \delta: 164.3$, $149.1,145.2,142.7,139.1,137.6,137.1,135.3$ (q, $J=34.0$ $\mathrm{Hz}), 133.5,133.5,130.6,123.0,128.7,127.4$ (q, $J=4.0$ $\mathrm{Hz}), 127.2,126.2$ (q, $J=4.0 \mathrm{~Hz}), 126.1,125.7,122.7$, 122.9 (q, $J=272 \mathrm{~Hz}), 117.2,21.7 ;{ }^{19} \mathrm{~F}$ NMR $(376 \mathrm{MHz}$, $\left.\mathrm{CDCl}_{3}\right) \delta:-63.22$; IR (neat) $v: 3305,2921,1672,1523$, 1482, 1311, 1252, 1156, 1113, 1079, 713, $589 \mathrm{~cm}^{-1}$; HRMS (ESI-TOF) calcd for $\mathrm{C}_{24} \mathrm{H}_{17}-\mathrm{N}_{2} \mathrm{O}_{3} \mathrm{~F}_{3} \mathrm{SCl}[\mathrm{M}+\mathrm{H}]^{+}$ 505.0600, found 505.0589.

$N$-(5-氯-8-喹啉基)-4-氟-2-甲苯磺酰基苯甲酰胺(3f): 白色固体, 收率 70\%. m.p. 172.2 174.4 ${ }^{\circ} \mathrm{C} ;{ }^{1} \mathrm{H}$ NMR $\left(400 \mathrm{MHz}, \mathrm{CDCl}_{3}\right) \delta: 10.05(\mathrm{~s}, 1 \mathrm{H}), 8.79$ (d, $J=7.0 \mathrm{~Hz}$, 2H), 8.59 (d, $J=8.4 \mathrm{~Hz}, 1 \mathrm{H}), 7.91$ (d, $J=8.2 \mathrm{~Hz}, 1 \mathrm{H}), 7.85$ $(\mathrm{d}, J=8.0 \mathrm{~Hz}, 2 \mathrm{H}), 7.72 \sim 7.61(\mathrm{~m}, 2 \mathrm{H}), 7.62 \sim 7.50(\mathrm{~m}$, $1 \mathrm{H}), 7.35$ (t, $J=8.2 \mathrm{~Hz}, 1 \mathrm{H}), 7.23$ (d, $J=8.0 \mathrm{~Hz}, 2 \mathrm{H}), 2.34$ $(\mathrm{s}, 3 \mathrm{H}) ;{ }^{13} \mathrm{C}$ NMR $\left(100 \mathrm{MHz}, \mathrm{CDCl}_{3}\right) \delta: 164.9,162.8(\mathrm{~d}$, $J=253.0 \mathrm{~Hz}$ ), 149.0, 145.0, 141.7 (d, $J=7.10 \mathrm{~Hz}), 139.1$, 137.3, 133.6, 133.5, 133.2, 131.2 (d, $J=6.0 \mathrm{~Hz}), 129.9$,
128.7, 127.3, 126.1, 125.4, 122.6, 120.6 (d, $J=21.0 \mathrm{~Hz})$, $117.2(\mathrm{~d}, J=25.0 \mathrm{~Hz}), 117.1,21.8 ;{ }^{19} \mathrm{~F}$ NMR (376 MHz, $\left.\mathrm{CDCl}_{3}\right) \delta:-106.79$; IR (neat) $v: 3318,2920,2341,1685$, 1520, 1473, 1320, 1149, 703, 655, $575 \mathrm{~cm}^{-1}$; HRMS (ESI-TOF) calcd for $\mathrm{C}_{25} \mathrm{H}_{22} \mathrm{~N}_{2} \mathrm{O}_{3} \mathrm{SCl}[\mathrm{M}+\mathrm{H}]^{+}$465.1034, found 465.1031 .

$N$-(5-氯-8-喹啉基)-4-氯-2-甲苯磺酰基苯甲酰胺 (3g): 黄褐色固体，收率 51\%. m.p. 220.9 202.3 ${ }^{\circ} \mathrm{C} ;{ }^{1} \mathrm{H}$ NMR (400 MHz, $\left.\mathrm{CDCl}_{3}\right) \delta: 10.05(\mathrm{~s}, 1 \mathrm{H}), 8.82 \sim 8.74(\mathrm{~m}$, 2H), 8.59 (dd, $J=8.4,1.6 \mathrm{~Hz}, 1 \mathrm{H}), 8.19$ (d, $J=2.0 \mathrm{~Hz}$, $1 \mathrm{H}), 7.90 \sim 7.84(\mathrm{~m}, 2 \mathrm{H}), 7.67(\mathrm{~d}, J=8.4 \mathrm{~Hz}, 1 \mathrm{H}), 7.62 \sim$ $7.53(\mathrm{~m}, 3 \mathrm{H}), 7.23(\mathrm{~d}, J=8.0 \mathrm{~Hz}, 2 \mathrm{H}), 2.34(\mathrm{~s}, 3 \mathrm{H}) ;{ }^{13} \mathrm{C}$ NMR $\left(100 \mathrm{MHz}, \mathrm{CDCl}_{3}\right) \delta: 164.8,149.0,145.0,141.0$, $139.1,136.8,135.2$, 133.6, 133.6, 133.5, 130.3, 129.9, $129.8,128.7,127.3,127.2,126.1,125.4,122.6,117.1$, 21.8; IR (neat) v: 3316, 2921, 1689, 1521, 1471, 1317, 1157, 1088, 896, 678, 653, $572 \mathrm{~cm}^{-1}$; HRMS (ESI-TOF) calcd for $\mathrm{C}_{23} \mathrm{H}_{17} \mathrm{~N}_{2} \mathrm{O}_{3} \mathrm{SCl}_{2}[\mathrm{M}+\mathrm{H}]^{+}$471.0331, found 471.0322 .

$N$-(5-氯-8-喹啉基)-4-甲基-2-甲苯磺酰基苯甲酰胺 (3h): 白色固体，收率 54\%. m.p. 209.6 210.3 ${ }^{\circ} \mathrm{C} ;{ }^{1} \mathrm{H}$ NMR (400 MHz, $\left.\mathrm{CDCl}_{3}\right) \delta: 10.01(\mathrm{~s}, 1 \mathrm{H}), 8.81(\mathrm{~d}, J=$ $8.4 \mathrm{~Hz}, 1 \mathrm{H}), 8.78(\mathrm{~d}, J=4.0 \mathrm{~Hz}, 1 \mathrm{H}), 8.58$ (dd, $J=8.8$, $1.5 \mathrm{~Hz}, 1 \mathrm{H}), 8.02(\mathrm{~d}, J=1.6 \mathrm{~Hz}, 1 \mathrm{H}), 7.86(\mathrm{~d}, J=8.0$ $\mathrm{Hz}, 2 \mathrm{H}), 7.67$ (d, $J=8.4 \mathrm{~Hz}, 1 \mathrm{H}), 7.56$ (dd, $J=8.4,4.2$ $\mathrm{Hz}, 1 \mathrm{H}), 7.53$ (d, $J=7.6 \mathrm{~Hz}, 1 \mathrm{H}), 7.46$ (d, $J=7.6 \mathrm{~Hz}$, $1 \mathrm{H}), 7.21$ (d, J=8.0 Hz, 2H), 2.49 (s, 3H), 2.32 (s, 3H); ${ }^{13} \mathrm{C}$ NMR $\left(100 \mathrm{MHz}, \mathrm{CDCl}_{3}\right) \delta: 166.0,148.9,144.3$, $141.2,139.2,139.0,138.3,134.4,134.1,133.9,133.4$, $130.1,129.7,128.9,128.4,127.3,126.1,125.1,122.5$, 117.0, 29.4, 21.5; IR (neat) $v: 3333,2920,1682,1518$, 1475, 1314, 1292, 1145, 705, 653, 577, $542 \mathrm{~cm}^{-1}$; HRMS (ESI-TOF) calcd for $\mathrm{C}_{24} \mathrm{H}_{20} \mathrm{~N}_{2} \mathrm{O}_{3} \mathrm{SCl}[\mathrm{M}+\mathrm{H}]^{+}$ 451.0878 , found 451.0879 .

$N$-(5-氯-8-喹啉基)-4-乙基-2-甲苯磺酰基苯甲酰胺 (3i): 白色固体，收率 51\%. m.p. $180.4 \sim 181.5{ }^{\circ} \mathrm{C} ;{ }^{1} \mathrm{H}$ NMR (400 MHz, $\left.\mathrm{CDCl}_{3}\right) \delta: 10.02(\mathrm{~s}, 1 \mathrm{H}), 8.79$ (dd, $J=$ $12.8,6.4 \mathrm{~Hz}, 2 \mathrm{H}), 8.58(\mathrm{~d}, J=8.4 \mathrm{~Hz}, 1 \mathrm{H}), 8.04(\mathrm{~s}, 1 \mathrm{H})$, $7.85(\mathrm{~d}, J=8.0 \mathrm{~Hz}, 2 \mathrm{H}), 7.66(\mathrm{~d}, J=8.4 \mathrm{~Hz}, 1 \mathrm{H}), 7.62 \sim$ $7.52(\mathrm{~m}, 2 \mathrm{H}), 7.49$ (d, $J=8.0 \mathrm{~Hz}, 1 \mathrm{H}), 7.20$ (d, $J=8.0 \mathrm{~Hz}$, 2H), 2.78 (q, $J=7.6 \mathrm{~Hz}, 2 \mathrm{H}), 2.31$ (s, 3H), 1.30 (s, 3H); ${ }^{13} \mathrm{C} \mathrm{NMR}\left(100 \mathrm{MHz}, \mathrm{CDCl}_{3}\right) \delta: 166.0,148.9,147.4,144.4$, $139.2,139.0,138.2,134.6,133.9,133.4,133.0,129.7$, $129.1,129.0,128.4,127.3,126.1,125.1,122.5,117.0$, 28.8, 21.7, 15.3; IR (neat) $v: 3330,2920,1680,1518$, 
1475, 1316, 1297, 1145, 940, 656, 576, $559 \mathrm{~cm}^{-1}$; HRMS (ESI-TOF) calcd for $\mathrm{C}_{27} \mathrm{H}_{20} \mathrm{~N}_{2} \mathrm{O}_{3} \mathrm{SCl}[\mathrm{M}+\mathrm{H}]^{+} 487.0878$, found 487.08720 .

$N$-(5-氯-8-喹啉基)-4-(叔丁基)-2-甲苯磺酰基苯甲酰 胺(3j): 黄色固体, 收率 50\%. m.p. 201.5 203.6 ${ }^{\circ} \mathrm{C} ;{ }^{1} \mathrm{H}$ NMR (400 MHz, $\left.\mathrm{CDCl}_{3}\right) \delta: 10.04(\mathrm{~s}, 1 \mathrm{H}), 8.80$ (d, $J=8.4$ $\mathrm{Hz}, 1 \mathrm{H}), 8.76$ (dd, $J=4.4,1.6 \mathrm{~Hz}, 1 \mathrm{H}), 8.55$ (dt, $J=8.4$, $1.6 \mathrm{~Hz}, 1 \mathrm{H}), 8.24(\mathrm{~d}, J=2.0 \mathrm{~Hz}, 1 \mathrm{H}), 7.84(\mathrm{~d}, J=8.0 \mathrm{~Hz}$, $2 \mathrm{H}), 7.72 \sim 7.61(\mathrm{~m}, 2 \mathrm{H}), 7.61 \sim 7.45(\mathrm{~m}, 2 \mathrm{H}), 7.19(\mathrm{~d}, J=$ $8.0 \mathrm{~Hz}, 2 \mathrm{H}), 2.30(\mathrm{~s}, 3 \mathrm{H}), 1.38(\mathrm{~s}, 9 \mathrm{H}) ;{ }^{13} \mathrm{C}$ NMR (100 $\left.\mathrm{MHz}, \mathrm{CDCl}_{3}\right) \delta: 166.0,154.3,148.9,144.3,139.1,138.7$, $138.2,134.3,133.8,133.4,130.6,129.7,128.8,128.4$, 127.2, 126.6, 126.0, 125.0, 122.5, 117.0, 35.4, 31.1, 21.7; IR (neat) $v: 2921,1675,1520,1478,1315,1147,939,787$, 682, 656, 578, $558 \mathrm{~cm}^{-1}$; HRMS (ESI-TOF) calcd for $\mathrm{C}_{27} \mathrm{H}_{26} \mathrm{~N}_{2} \mathrm{O}_{3} \mathrm{SCl}[\mathrm{M}+\mathrm{H}]^{+}$493.1347, found 493.1347.

$N$-(5-氯-8-喹啉基)-2,4-二甲基-6-甲苯磺酰基苯甲酰 胺 $(3 \mathrm{k})$ : 淡黄色固体, 收率 78\%. m.p. 209.9 211.3 ${ }^{\circ} \mathrm{C}$; ${ }^{1} \mathrm{H}$ NMR (400 MHz, $\left.\mathrm{CDCl}_{3}\right) \delta: 9.84(\mathrm{~s}, 1 \mathrm{H}), 8.89$ (d, $J=$ $8.4 \mathrm{~Hz}, 1 \mathrm{H}), 8.72$ (dd, $J=4.4,1.6 \mathrm{~Hz}, 1 \mathrm{H}), 8.55$ (dd, $J=$ 8.4, $1.6 \mathrm{~Hz}, 1 \mathrm{H}), 7.86 \sim 7.78(\mathrm{~m}, 3 \mathrm{H}), 7.68(\mathrm{~d}, J=8.4 \mathrm{~Hz}$, $1 \mathrm{H}), 7.53(\mathrm{dd}, J=8.4,4.0 \mathrm{~Hz}, 1 \mathrm{H}), 7.28(\mathrm{~s}, 1 \mathrm{H}), 7.17$ (d, $J=8.0 \mathrm{~Hz}, 2 \mathrm{H}), 2.41$ (s, 3H), 2.39 (s, 3H), 2.30 (s, 3H); ${ }^{13} \mathrm{C}$ NMR $\left(100 \mathrm{MHz}, \mathrm{CDCl}_{3}\right) \delta: 165.9,148.8,144.2,140.1$, $139.1,138.7,138.5,137.1,136.2,133.8,133.6,133.4$, $129.7,128.2,127.4,127.3,126.0,124.9,122.5,116.9$, 21.7, 21.3, 19.3; IR (neat) $v: 3328,2920,1675,1517$, 1479, 1314, 1139, 1041, 663, 585, $564 \mathrm{~cm}^{-1}$; HRMS (ESI-TOF) calcd for $\mathrm{C}_{21} \mathrm{H}_{21} \mathrm{~N}_{2} \mathrm{O}_{2} \mathrm{~S}[\mathrm{M}+\mathrm{H}]^{+}$305.1536, found 305.1532 .

$N$-(5-氯-8-喹啉基)-6-甲苯磺酰基-2,3-二氢苯并 $[b][1,4]$ 二噁英-5-羧酰胺(3I): 白色固体, 收率 71\%. m.p. $225.6 \sim 228.6{ }^{\circ} \mathrm{C} ;{ }^{1} \mathrm{H}$ NMR $\left(400 \mathrm{MHz}, \mathrm{CDCl}_{3}\right) \delta: 10.03 \sim$ $9.86(\mathrm{~m}, 1 \mathrm{H}), 8.90(\mathrm{~d}, J=8.4 \mathrm{~Hz}, 1 \mathrm{H}), 8.77$ (dd, $J=4.4$, $1.6 \mathrm{~Hz}, 1 \mathrm{H}), 8.58(\mathrm{dd}, J=8.4,1.6 \mathrm{~Hz}, 1 \mathrm{H}), 7.89 \sim 7.76(\mathrm{~m}$, 2H), 7.67 (dd, $J=8.4,7.2 \mathrm{~Hz}, 2 \mathrm{H}), 7.56$ (dd, $J=8.4,4.0$ $\mathrm{Hz}, 1 \mathrm{H}), 7.17$ (d, $J=8.0 \mathrm{~Hz}, 2 \mathrm{H}), 7.04$ (d, $J=8.8 \mathrm{~Hz}, 1 \mathrm{H})$, $4.38 \sim 4.17(\mathrm{~m}, 4 \mathrm{H}), 2.31(\mathrm{~s}, 3 \mathrm{H}) ;{ }^{13} \mathrm{C}$ NMR $(100 \mathrm{MHz}$, $\left.\mathrm{CDCl}_{3}\right) \delta: 165.4,148.9,144.6,139.1,138.4,135.5,135.4$, $135.2,133.8,133.4,130.7,129.9,129.4,128.7,128.3$, $127.4,126.7,126.2,125.3,124.0,122.6,117.1,21.7$; IR (neat) $v: 2922,1678,1519,1476,1288,1258,1150,1079$, 788, 656, 582, $524 \mathrm{~cm}^{-1}$; HRMS (ESI-TOF) calcd for $\mathrm{C}_{25} \mathrm{H}_{20} \mathrm{~N}_{2} \mathrm{O}_{5} \mathrm{SCl}[\mathrm{M}+\mathrm{H}]^{+}$495.0776, found 495.0773.

$N$-(5-氯-8-喹啉基)-2-甲苯磺酰基-1-菜酰胺 $(\mathbf{3 m})$ : 淡
黄色固体, 收率 46\%. m.p. 214.9 217.6 ${ }^{\circ} \mathrm{C}$ (lit. ${ }^{[16] \text { : }}$ $220 \sim 223{ }^{\circ} \mathrm{C}$ ); ${ }^{1} \mathrm{H}$ NMR (400 MHz, $\mathrm{CDCl}_{3}$ ) $\delta: 10.14$ (s, $1 \mathrm{H}), 9.06$ (d, $J=8.4 \mathrm{~Hz}, 1 \mathrm{H}), 8.69$ (dd, $J=4.4,1.6 \mathrm{~Hz}$, $1 \mathrm{H}), 8.58(\mathrm{dd}, J=8.4,1.6 \mathrm{~Hz}, 1 \mathrm{H}), 8.15 \sim 8.05(\mathrm{~m}, 2 \mathrm{H})$, $8.03(\mathrm{~d}, J=8.8 \mathrm{~Hz}, 1 \mathrm{H}), 7.92(\mathrm{dd}, J=12.4,8.0 \mathrm{~Hz}, 3 \mathrm{H})$, $7.74(\mathrm{~d}, J=8.4 \mathrm{~Hz}, 1 \mathrm{H}), 7.64(\mathrm{t}, J=7.6 \mathrm{~Hz}, 1 \mathrm{H}), 7.60 \sim$ 7.49 (m, 2H), 7.21 (d, J=8.0 Hz, 2H), $2.33(\mathrm{~s}, 3 \mathrm{H}) ;{ }^{13} \mathrm{C}$ NMR (100 MHz, $\left.\mathrm{CDCl}_{3}\right) \delta: 165.4,148.9,144.5,139.1$, $138.4,138.3,135.5,135.4,135.2,133.8,133.4,130.7$, $130.1,129.9,129.4,128.7,128.3,127.4,126.7,126.1$, 125.3, 124.0, 122.6, 117.1, 21.7; IR (neat) $v: 2921,1677$, $1517,1477,1317,1152,901,811,684,663,543,467$, $\mathrm{cm}^{-1}$; HRMS (ESI-TOF) calcd for $\mathrm{C}_{21} \mathrm{H}_{21} \mathrm{~N}_{2} \mathrm{O}_{2} \mathrm{~S}[\mathrm{M}+\mathrm{H}]^{+}$ 305.1536 , found 305.1532 .

$N$-(5-氯-8-喹啉基)-2-甲基-6-(苯磺酰基)苯甲酰胺 (4a): 黄色固体, 收率 64\%. m.p. 226.0 228.5 ${ }^{\circ} \mathrm{C} ;{ }^{1} \mathrm{H}$ NMR (400 MHz, $\left.\mathrm{CDCl}_{3}\right) \delta: 9.90(\mathrm{~s}, 1 \mathrm{H}), 8.89$ (d, $J=8.4$ $\mathrm{Hz}, 1 \mathrm{H}), 8.76$ (dd, $J=4.4,1.6 \mathrm{~Hz}, 1 \mathrm{H}), 8.58$ (dd, $J=8.4$, $1.6 \mathrm{~Hz}, 1 \mathrm{H}), 8.02$ (t, $J=4.8 \mathrm{~Hz}, 1 \mathrm{H}), 8.00 \sim 7.91(\mathrm{~m}, 2 \mathrm{H})$, $7.69(\mathrm{~d}, J=8.4 \mathrm{~Hz}, 1 \mathrm{H}), 7.56(\mathrm{dd}, J=8.4,4.2 \mathrm{~Hz}, 1 \mathrm{H})$, $7.51(\mathrm{~d}, J=4.8 \mathrm{~Hz}, 2 \mathrm{H}), 7.40(\mathrm{t}, J=7.8 \mathrm{~Hz}, 2 \mathrm{H}), 2.45(\mathrm{~s}$, $3 \mathrm{H}) ;{ }^{13} \mathrm{C}$ NMR $\left(100 \mathrm{MHz}, \mathrm{CDCl}_{3}\right) \delta: 165.6,148.9,141.4$, 139.2 , 138.6, 137.38, 136.4, 136.2, 135.8, 133.7, 133.5, $133.4,129.8,129.1,128.2,127.4,126.2,125.2,122.6$, 117.1, 19.4; IR (neat) $v: 3342,2920,1680,1518,1478$, 1317, 1139, 1081, 682, 601, $579 \mathrm{~cm}^{-1}$; HRMS (ESI-TOF) calcd for $\mathrm{C}_{23} \mathrm{H}_{18} \mathrm{~N}_{2} \mathrm{O}_{3} \mathrm{SCl}[\mathrm{M}+\mathrm{H}]^{+} 437.0821$, found 437.0719 .

$N$-(5-氯-8-喹啉基)-2-((4-氟苯基)磺酰基)-6-甲基苯 甲酰胺 (4b): 白色固体，收率 61\%. m.p. 210.7 $212.9{ }^{\circ} \mathrm{C} ;{ }^{1} \mathrm{H}$ NMR (400 MHz, $\left.\mathrm{CDCl}_{3}\right) \delta$ : $9.91(\mathrm{~s}, 1 \mathrm{H})$, 8.89 (d, $J=8.4 \mathrm{~Hz}, 1 \mathrm{H}), 8.77$ (dd, $J=4.3,1.6 \mathrm{~Hz}, 1 \mathrm{H})$, $8.59(\mathrm{dd}, J=8.4,1.6 \mathrm{~Hz}, 1 \mathrm{H}), 8.00(\mathrm{td}, J=8.8,8.4,4.8 \mathrm{~Hz}$, $3 \mathrm{H}), 7.70(\mathrm{~d}, J=8.4 \mathrm{~Hz}, 1 \mathrm{H}), 7.57$ (dd, $J=8.6,4.2 \mathrm{~Hz}$, $1 \mathrm{H}), 7.55 \sim 7.45(\mathrm{~m}, 2 \mathrm{H}), 7.08(\mathrm{t}, J=8.5 \mathrm{~Hz}, 2 \mathrm{H}), 2.45(\mathrm{~s}$, $3 \mathrm{H}) ;{ }^{13} \mathrm{C} \mathrm{NMR}\left(100 \mathrm{MHz}, \mathrm{CDCl}_{3}\right) \delta: 165.7,165.6(\mathrm{~d}, J=$ $255.1 \mathrm{~Hz}), 149.0,139.2,138.5,137.4$ (d, $J=7.1 \mathrm{~Hz}), 136.3$ (d, $J=6.1 \mathrm{~Hz}), 136.0,133.6,133.6,131.3,131.2,123.0$, $127.4,127.3,126.2,125.3,122.6,117.0,116.5$ (d, $J=23.0$ $\mathrm{Hz}), 19.5 ;{ }^{19} \mathrm{~F}$ NMR $\left(376 \mathrm{MHz}, \mathrm{CDCl}_{3}\right) \delta$ : -104.13 ; IR (neat) $v: 3314,2922,1682,1518,1477,1319,1139,1080$, 834, 663, 590, $558 \mathrm{~cm}^{-1}$; HRMS (ESI-TOF) calcd for $\mathrm{C}_{23} \mathrm{H}_{17} \mathrm{~N}_{2} \mathrm{O}_{3} \mathrm{SCl}[\mathrm{M}+\mathrm{H}]^{+}$455.0627, found 455.0627.

$N$-(5-氯-8-喹啉基)-2-((4-氯苯基)磺酰基)-6-甲基苯 甲酰胺 (4c): 白色固体，收率 64\%. m.p. 192.9 
$194.9{ }^{\circ} \mathrm{C} ;{ }^{1} \mathrm{H}$ NMR $\left(400 \mathrm{MHz}, \mathrm{CDCl}_{3}\right) \delta: 9.86(\mathrm{~s}, 1 \mathrm{H})$, $8.89(\mathrm{~d}, J=8.4 \mathrm{~Hz}, 1 \mathrm{H}), 8.81 \sim 8.68(\mathrm{~m}, 1 \mathrm{H}), 8.64 \sim 8.50$ (m, $1 \mathrm{H}), 8.02(\mathrm{t}, J=4.8 \mathrm{~Hz}, 1 \mathrm{H}), 7.94 \sim 7.82(\mathrm{~m}, 2 \mathrm{H}), 7.70$ (d, $J=8.4 \mathrm{~Hz}, 1 \mathrm{H}), 7.57(\mathrm{dd}, J=8.4,4.2 \mathrm{~Hz}, 1 \mathrm{H}), 7.53(\mathrm{~d}$, $J=4.0 \mathrm{~Hz}, 2 \mathrm{H}), 7.35$ (d, $J=8.0 \mathrm{~Hz}, 2 \mathrm{H}), 2.45$ (s, $3 \mathrm{H}) ;{ }^{13} \mathrm{C}$ NMR $\left(100 \mathrm{MHz}, \mathrm{CDCl}_{3}\right) \delta: 165.5,149.0,140.1,139.9$, $139.1,138.2,137.5,136.4,136.1,133.5,129.9,129.7$, 129.4, 127.4, 126.1, 125.3, 122.6, 117.0, 19.4; IR (neat) $v$ : 3311, 2920, 1682, 1518, 1476, 1320, 1085, 938, 756, 621, $584,558 \mathrm{~cm}^{-1}$; HRMS (ESI-TOF) calcd for $\mathrm{C}_{23} \mathrm{H}_{17} \mathrm{~N}_{2}$ $\mathrm{O}_{3} \mathrm{SCl}_{2}[\mathrm{M}+\mathrm{H}]^{+}$471.0331, found 471.0320.

$\mathrm{N}$-(5-氯-8-喹啉基)-2-((4-溴苯基)磺酰基)-6-甲基苯 甲酰胺 (4d): 白色固体, 收率 62\%. m.p. 188.5 $190.3{ }^{\circ} \mathrm{C} ;{ }^{1} \mathrm{H}$ NMR $\left(400 \mathrm{MHz}, \mathrm{CDCl}_{3}\right) \delta: 9.85$ (s, 1H), $8.89(\mathrm{~d}, J=8.4 \mathrm{~Hz}, 1 \mathrm{H}), 8.73$ (dd, $J=4.0,1.6 \mathrm{~Hz}, 1 \mathrm{H})$, 8.55 (dd, $J=8.4,1.7 \mathrm{~Hz}, 1 \mathrm{H}), 8.01$ (t, $J=4.4 \mathrm{~Hz}, 1 \mathrm{H}), 7.81$ (d, $J=8.4 \mathrm{~Hz}, 2 \mathrm{H}), 7.69$ (d, $J=8.4 \mathrm{~Hz}, 1 \mathrm{H}), 7.58 \sim 7.46$ (m, 5H), $2.44(\mathrm{~s}, 3 \mathrm{H}) ;{ }^{13} \mathrm{C}$ NMR $\left(100 \mathrm{MHz}, \mathrm{CDCl}_{3}\right) \delta$ : $165.4,149.0,140.4,138.9,138.0,137.4,136.3,136.1$, $133.5,133.4,132.4,129.9,129.7,128.6,127.3,127.3$, 126.0, 125.2, 122.6, 116.9, 19.4; IR (neat) $v: 3311,2920$, $1682,1518,1476,1320,1085,938,756,621,584,558$ $\mathrm{cm}^{-1}$; HRMS (ESI-TOF) calcd for $\mathrm{C}_{23} \mathrm{H}_{17} \mathrm{~N}_{2} \mathrm{O}_{3} \mathrm{SCl}_{2}[\mathrm{M}+$ $\mathrm{H}^{+}$471.0331, found 471.0320.

$\mathrm{N}$-(5-氯-8-喹啉基)-2-甲基-6-((4-(三氟甲基)苯基)磺 酰基)苯甲酰胺 (4e): 白色固体, 收率 78\%. m.p. 176.9 $178.5{ }^{\circ} \mathrm{C} ;{ }^{1} \mathrm{H}$ NMR $\left(400 \mathrm{MHz}, \mathrm{CDCl}_{3}\right) \delta: 9.88(\mathrm{~s}, 1 \mathrm{H})$, $8.89(\mathrm{~d}, J=8.4 \mathrm{~Hz}, 1 \mathrm{H}), 8.74(\mathrm{dd}, J=4.4,1.7 \mathrm{~Hz}, 1 \mathrm{H})$, $8.59(\mathrm{dd}, J=8.4,1.7 \mathrm{~Hz}, 1 \mathrm{H}), 8.10(\mathrm{~d}, J=8.0 \mathrm{~Hz}, 2 \mathrm{H})$, $8.06(\mathrm{dd}, J=6,3.4 \mathrm{~Hz}, 1 \mathrm{H}), 7.71(\mathrm{~d}, J=8.4 \mathrm{~Hz}, 1 \mathrm{H}), 7.66$ (d, $J=8.2 \mathrm{~Hz}, 2 \mathrm{H}), 7.60 \sim 7.49(\mathrm{~m}, 3 \mathrm{H}), 2.46(\mathrm{~s}, 3 \mathrm{H}) ;{ }^{13} \mathrm{C}$ NMR $\left(100 \mathrm{MHz}, \mathrm{CDCl}_{3}\right) \delta: 165.4,149.1,144.9,139.0$, 137.6, 136.57, 134.9 (q, $J=33.0 \mathrm{~Hz}), 133.6,133.5,133.4$, $130.0,128.8,128.8,128.7,127.6$ (q, $J=4.0 \mathrm{~Hz}), 127.3$ (q, $J=5.0 \mathrm{~Hz}), 125.4,123.2(\mathrm{q}, J=271.0 \mathrm{~Hz}), 122.7,122.6$, 117.0, 19.4; ${ }^{19} \mathrm{~F}$ NMR (376 MHz, $\left.\mathrm{CDCl}_{3}\right) \delta:-63.21$; IR (neat) $v$ : 2922, 1591, 1518, 1479, 1319, 1163, 1128, 1060, 709, 616, 598, $555 \mathrm{~cm}^{-1}$; HRMS (ESI-TOF) calcd for $\mathrm{C}_{24} \mathrm{H}_{17} \mathrm{~N}_{2} \mathrm{O}_{3} \mathrm{~F}_{3} \mathrm{SCl}[\mathrm{M}+\mathrm{H}]^{+}$505.0600, found 505.0591.

$\mathrm{N}$-(5-氯-8-喹啉基)-2-((4-甲氧基苯基)磺酰基)-6-甲 基苯甲酰胺(4f): 白色固体, 收率 66\%. m.p. 89.1 $91.5{ }^{\circ} \mathrm{C} ;{ }^{1} \mathrm{H}$ NMR $\left(400 \mathrm{MHz}, \mathrm{CDCl}_{3}\right) \delta$ : $9.87(\mathrm{~s}, 1 \mathrm{H}), 8.90$ (d, $J=8.4 \mathrm{~Hz}, 1 \mathrm{H}), 8.75(\mathrm{dd}, J=4.2,1.6 \mathrm{~Hz}, 1 \mathrm{H}), 8.57$ (dd, $J=8.4,1.7 \mathrm{~Hz}, 1 \mathrm{H}), 8.07 \sim 7.95(\mathrm{~m}, 1 \mathrm{H}), 7.92 \sim 7.81$ (m, 2H), 7.69 (d, $J=8.4 \mathrm{~Hz}, 1 \mathrm{H}), 7.55$ (dd, $J=8.4,4.2 \mathrm{~Hz}$,
1H), 7.49 (d, $J=2.8 \mathrm{~Hz}, 1 \mathrm{H}), 7.48(\mathrm{~s}, 1 \mathrm{H}), 6.88 \sim 6.79(\mathrm{~m}$, $2 \mathrm{H}), 3.75(\mathrm{~s}, 3 \mathrm{H}), 2.43(\mathrm{~s}, 3 \mathrm{H}) ;{ }^{13} \mathrm{C}$ NMR $(100 \mathrm{MHz}$, $\left.\mathrm{CDCl}_{3}\right) \delta: 165.7,163.4,148.9,139.2,139.0,137.2,136.0$, $135.4,133.7,133.4,132.8,130.5,129.7,127.3,127.0$, 126.0, 125.1, 122.5, 117.0, 114.3, 55.6, 19. 4; IR (neat) $v$ : 3336, 2920, 1678, 1517, 1478, 1318, 1259, 1137, 1083, $665,591,560 \mathrm{~cm}^{-1}$; HRMS (ESI-TOF) calcd for $\mathrm{C}_{24} \mathrm{H}_{20^{-}}$ $\mathrm{N}_{2} \mathrm{O}_{4} \mathrm{SCl}[\mathrm{M}+\mathrm{H}]^{+}$467.0827, found 467.0827.

$N$-(5-氯-8-喹啉基)-2-((4-(叔丁基)苯基)磺酰基)-6甲基苯甲酰胺 $(\mathbf{4 g})$ : 黄色粘稠油状物, 收率 $61 \%$. ${ }^{1} \mathrm{H}$ NMR (400 MHz, $\left.\mathrm{CDCl}_{3}\right) \delta: 9.91(\mathrm{~s}, 1 \mathrm{H}), 8.92(\mathrm{~d}, J=8.4$ $\mathrm{Hz}, 1 \mathrm{H}), 8.79 \sim 8.65(\mathrm{~m}, 1 \mathrm{H}), 8.59 \sim 8.46(\mathrm{~m}, 1 \mathrm{H}), 8.00$ (dd, $J=6.6,2.8 \mathrm{~Hz}, 1 \mathrm{H}), 7.91(\mathrm{~d}, J=8.2 \mathrm{~Hz}, 2 \mathrm{H}), 7.69$ (d, $J=8.4 \mathrm{~Hz}, 1 \mathrm{H}), 7.53(\mathrm{dd}, J=8.4,4.2 \mathrm{~Hz}, 1 \mathrm{H}), 7.50 \sim 7.44$ (m, 2H), $7.45 \sim 7.34(\mathrm{~m}, 2 \mathrm{H}), 2.43(\mathrm{~s}, 3 \mathrm{H}), 1.25(\mathrm{~s}, 9 \mathrm{H})$; ${ }^{13} \mathrm{C}$ NMR (100 MHz, $\left.\mathrm{CDCl}_{3}\right) \delta: 165.7,157.1,148.9,139.0$, $138.9,138.28,137.1,136.1,135.5,133.6,133.3,129.8$, $128.1,127.2,126.2,126.0,125.0,122.5,117.0,35.2,31.0$, 19.4; IR (neat) v: 3338, 2962, 1679, 1516, 1478, 1318, 1142, 938, 733, 630, 590, $568 \mathrm{~cm}^{-1}$; HRMS (ESI-TOF) calcd for $\mathrm{C}_{27} \mathrm{H}_{26} \mathrm{~N}_{2} \mathrm{O}_{3} \mathrm{SCl}[\mathrm{M}+\mathrm{H}]^{+}$493.1347, found 493.1347.

$N$-(5-氯-8-喹啉基)-2-甲基-6-(荎基-2-磺酰基)苯甲 酰胺 (4h): 淡黄色固体, 收率 79\%. m.p. $224.5 \sim$ $226.1{ }^{\circ} \mathrm{C} ;{ }^{1} \mathrm{H}$ NMR $\left(400 \mathrm{MHz}, \mathrm{CDCl}_{3}\right) \delta$ : $9.70(\mathrm{~s}, 1 \mathrm{H})$, $8.89(\mathrm{~d}, J=8.4 \mathrm{~Hz}, 1 \mathrm{H}), 8.51(\mathrm{dd}, J=8.4,1.8 \mathrm{~Hz}, 1 \mathrm{H})$, $8.49 \sim 8.38(\mathrm{~m}, 2 \mathrm{H}), 8.15$ (dd, $J=7.4,2.0 \mathrm{~Hz}, 1 \mathrm{H}), 7.90 \sim$ $7.76(\mathrm{~m}, 2 \mathrm{H}), 7.72(\mathrm{~d}, J=8.0 \mathrm{~Hz}, 1 \mathrm{H}), 7.68(\mathrm{~d}, J=8.4 \mathrm{~Hz}$, 1H), $7.59 \sim 7.53(\mathrm{~m}, 2 \mathrm{H}), 7.53 \sim 7.49(\mathrm{~m}, 2 \mathrm{H}), 7.47 \sim 7.38$ (m, 2H), 2.43 (s, 3H); ${ }^{13} \mathrm{C}$ NMR $\left(100 \mathrm{MHz}, \mathrm{CDCl}_{3}\right) \delta$ : $165.2,148.7,139.5,138.8,138.4,138.0,137.6,136.4$, $135.9,135.1,133.5,133.4,132.1,129.8,129.8,129.5$, $129.4,129.0,127.8,127.4,127.4,126.0,125.2,122.9$, 122.5, 117.1, 19.4; IR (neat) $v: 3342,2920,1679,1517$, 1478, 1316, 1259, 1120, 1069, 788, 656, $557 \mathrm{~cm}^{-1}$; HRMS (ESI-TOF) calcd for $\mathrm{C}_{27} \mathrm{H}_{20} \mathrm{~N}_{2} \mathrm{O}_{3} \mathrm{SCl}[\mathrm{M}+\mathrm{H}]^{+} 487.0878$, found 487.0868 .

\section{3 自由基捕获剂实验}

将 $3 \mathrm{~g}(0.5 \mathrm{mmol})$ 溶于四氢呋喃(THF) $(5 \mathrm{~mL})$ 中, $0{ }^{\circ} \mathrm{C}$ 条件下分批加入 $\mathrm{NaH}(1 \mathrm{mmol})$ 并摚拌 $1 \mathrm{~h}$. 随后在 $0{ }^{\circ} \mathrm{C}$ 下缓慢加入 MeI (2.5 mmol)搅拌 $1 \mathrm{~h}$, 然后升至室温 搅拌过夜. 加水淬灭反应后用 $\mathrm{Et}_{2} \mathrm{O}$ 萃取, 收集有机相并 用无水 $\mathrm{Na}_{2} \mathrm{SO}_{4}$ 干燥, 过滤, 减压浓缩, 得到黄色中间体 5 粗产品固体. 将中间体 5 和 $\mathrm{NaOH}(6 \mathrm{mmol})$ 溶解在 EtOH $(5 \mathrm{~mL})$ 中, $130{ }^{\circ} \mathrm{C}$ 搅拌 $24 \mathrm{~h}$. 反应完成后加水 $(20$ 
$\mathrm{mL}$ )萃取, 收集水相并用 $1 \mathrm{~mol} / \mathrm{L} \mathrm{HCl}$ 酸化, 随后用 EtOAc $(10 \mathrm{~mL} \times 3)$ 萃取并收集有机相用无水 $\mathrm{Na}_{2} \mathrm{SO}_{4}$ 干 燥, 过滤, 减压浓缩, 残余物通过硅胶柱层析(石油醚/ 乙酸乙酯, $V: V=3: 1$ )分离得到目标产物 6 .

4-氯-2-甲苯磺酰基苯甲酸 (6): 淡黄色固体, 收率 57\%. m.p. $145.2 \sim 147.5{ }^{\circ} \mathrm{C}$ (lit. ${ }^{[18]}$ m.p. $155{ }^{\circ} \mathrm{C}$ ); ${ }^{1} \mathrm{H}$ NMR (400 MHz, DMSO- $\left.d_{6}\right) \delta: 8.04(\mathrm{~d}, J=8.0 \mathrm{~Hz}, 2 \mathrm{H})$, $7.89(\mathrm{~d}, J=2.0 \mathrm{~Hz}, 1 \mathrm{H}), 7.62(\mathrm{dd}, J=8.4,2.0 \mathrm{~Hz}, 1 \mathrm{H})$, 7.39 (d, $J=8.2 \mathrm{~Hz}, 1 \mathrm{H}), 7.34$ (d, $J=8.0 \mathrm{~Hz}, 2 \mathrm{H}), 2.36$ (s, $3 \mathrm{H}) ;{ }^{13} \mathrm{C}$ NMR (101 MHz, DMSO- $\left.d_{6}\right) \delta: 169.27,143.67$, $138.72,137.83,132.96,130.92,130.47,129.20,128.48$, $127.79,125.53,21.08$.

\section{$3.4 \mathrm{AQ}^{\prime}$ 导向基团的脱除}

向干燥的反应管中依次加入搅拌子、 $\mathrm{DMF}(1 \mathrm{~mL})$ 、 1,1-二苯乙烯 $(72.1 \mathrm{mg}, 0.4 \mathrm{mmol})$ 、对甲苯亚磺酸钠(2) (71.3 mg, 0.4mmol)、醋酸铜 $(7.2 \mathrm{mg}, 0.02 \mathrm{mmol}) 、$ 苯甲酸 (7.4 mg, $0.03 \mathrm{mmol}$ )和特戊酸钾 $(56.0 \mathrm{mg}, 0.4 \mathrm{mmol})$, 将 反应体系置于 $80{ }^{\circ} \mathrm{C}$ 油浴中反应 $8 \mathrm{~h}$. 反应结束后冷却 至室温, 用乙酸乙酯 $(5 \mathrm{~mL})$ 稀释后，再用饱和食盐水 $(20$ $\mathrm{mL}$ )洗涤，合并有机相并用无水 $\mathrm{Na}_{2} \mathrm{SO}_{4}$ 干燥，过滤，减 压浓缩，通过 HRMS 检测到化合物 7 的生成. 2-甲苯磺 酰 基-1,1-二苯乙烯 (7): HRMS (ESI-TOF) calcd for $\mathrm{C}_{21} \mathrm{H}_{18} \mathrm{O}_{2} \mathrm{SNa}[\mathrm{M}+\mathrm{Na}]^{+}$357.0920, found 357.0920.

\section{辅助材料(Supporting Information) 化合物 3a $\sim 3 \mathrm{~m}$ 、} 4a $\sim 4 h$ 及 6 的 ${ }^{1} \mathrm{H} N M R 、{ }^{13} \mathrm{C} N M R 、{ }^{19} \mathrm{~F}$ NMR 谱图. 这 些材料可以免费从本刊网站(http://sioc-journal.cn/)上下 载.

\section{References}

[1] Patai, S.; Rappoport, C. Z.; Stirling, J. M. The Chemistry of Sulfones and Sulfoxides, Wiley, New York, 1988.

[2] Lopez de Compadre, R. L.; Pearlstein, R. A.; Hopfinger, A. J.; Seyde, J. K. J. Med. Chem. 1987, 30, 900.

[3] Iversen, P.; Tyrrell, C. J.; Kaisary, A. V.; Anderson, J. B.; Van Poppel, H. E. I. N.; Tammela, T. L.; Melezinek, I. J. Urol. 2000, 164, 1579 .

[4] Tanetani, Y.; Kaku, K.; Kawai, K.; Fujioka, T.; Shimizu, T. Pestic. Biochem. Physiol. 2009, 95, 47.

[5] Otzen, T.; Wempe, E. G.; Kunz, B.; Bartels, R.; Lehwark-Yvetot, G.; Hansel, W.; K. Schaper, J.; Seydel, J. K. J. Med. Chem. 2004, 47, 240.

[6] Simpkins, N. S. Sulphones in Organic Synthesis, Pergamon Press, Oxford, 1993.

[7] Ramberg, L.; B-cklund, B. Ark. Kemi, Mineral Geol. 1940, 27, 1.

[8] Julia, M.; Paris, J.-M. Tetrahedron Lett. 1973, 14, 4833.

[9] For recent examples, see: (a) Yuan, Z.; Wang, H.-Y.; Mu, X. Chen, P.; Guo, Y.-L.; Liu, G. J. Am. Chem. Soc. 2015, 137, 2468.

(b) Tang, X.; Huang, L.; Xu, Y.; Yang, J.; Wu, W.; Jiang, H. Angew. Chem., Int. Ed. 2014, 53, 4205.

(c) Xi, Y.; Dong, B.; McClain, E. J.; Wang, Q.; Gregg, T. L.; Akhmedov, N. G.; Petersen, J. L.; Shi, X. Angew. Chem., Int. Ed. 2014, 53, 4657 . (d) Handa, S.; Fennewald, J. C.; Lipshutz, B. H. Angew. Chem., Int. Ed. 2014, 53, 3432.

(e) Lu, Q.; Zhang, J.; Zhao, G.; Qi, Y.; Wang, H.; Lei, A. J. Am. Chem. Soc. 2013, 135, 11481.

(f) Liu, Q.; Zhang, J.; Wei, F.; Qi, Y.; Wang, H.; Liu, Z.; Lei, A. Angew. Chem., Int. Ed. 2013, 52, 7156.

(g) Yuan, G.; Zheng, J.; Gao, X.; Li, X.; Huang, L.; Chen, H.; Jiang, H. Chem. Commun. 2012, 48, 7513.

(h) Wu, X.-S.; Chen, Y.; Li, M.-B.; Zhou, M.-G.; Tian, S.-K. J. Am Chem. Soc. 2012, 134, 14694.

[10] Zhao, X.; Dimitrijević, E.; Dong, V. M. J. Am. Chem. Soc. 2009, $131,3466$.

[11] Liu, N.-W.; Liang, S.; Manolikakes, G. Synthesis 2016, 48, 1939. For selected reviews on transition-metal-catalyzed $\mathrm{C}-\mathrm{H}$ functionalization, see: (a) Giri, R.; Shi, B.-F.; Engle, K. M.; Maugel, N.; Yu, J.-Q. Chem. Soc. Rev. 2009, 38, 3242.

(b) Chen, X.; Engle, K. M.; Wang, D.-H.; Yu, J.-Q. Angew Chem., Int. Ed. 2009, 48, 5094.

(c) Lyons, T. W.; Sanford, M. S. Chem. Rev. 2010, 110, 1147.

(d) Arockiam, P. B.; Bruneau, C.; Dixneuf, P. H. Chem. Rev. 2012, 112,5879 .

(e) Ackermann, L. C. Acc. Chem. Res. 2014, 47, 281.

(f) Pei, P.; Zhang, F.; Yi, H.; Lei, A. Acta Chim. Sinica 2017, 75, 15 (in Chinese).

(裴朋昆, 张凡, 易红, 雷爱文, 化学学报, 2017, 75, 15.)

(g) Du, J.-Y.; Xia C.-G.; Sun, W. Acta Chim. Sinica 2018, 76, 329 (in Chinese)

(杜俊毅，夏春谷，孙伟，化学学报, 2018, 76, 329.)

For selected reviews on Directing-Group see: (h) Daugulis, O.; Do, H.-Q.; Shabashov, D. Acc. Chem. Res. 2009, 42, 1074.

(i) Li, D.-D.; He, C.-L.; Cai, H.-T.; Wang, G.-W. Chin. J. Org. Chem. 2013, 33, 203 (in Chinese).

(李丹丹, 何程林, 蔡海婷, 王官武, 有机化学, 2013, 33, 203.)

(j) Wang, S.; Yan, F.; Wang, L.-S.; Zhu, L. Chin. J. Org. Chem. 2018, 38, 291 (in Chinese).

(汪珊, 严泮, 汪连生, 朱磊, 有机化学, 2018, 38, 291.)

(k) Luo, F. Chin. J. Org. Chem. 2019, 39, 3084 (in Chinese)

(罗飞华, 有机化学, 2019, 39, 3084.)

For examples on Directing-Group, see: (e) Zhang, Q.; Yin, X.-S.; Zhao, S.; Fang, S.-L.; Shi, B.-F. Chem. Commun. 2014, 50, 8353.

(1) Yang, Q.-L.; Wang, X.-Y.; Wang, T.-L.; Yang, X.; Liu, D.; Tong,

X.; Wu, X.-Y.; Mei, T.-S. Org. Lett. 2019, 21, 2645.

(m) Yang, Q.-L.; Wang, X.-Y.; Weng, X.-J.; Yang, X.; Xu, X.-T.; Tong, X.; Fang, P.; Wu, X.-Y.; Mei, T.-S. Acta Chim. Sinica 2019, 77,866 (in Chinese).

(杨启亮，王向阳，翁信军，杨祥，徐学涛，童晓峰，方萍，伍新 燕，梅天胜，化学学报, 2019, 77, 866.)

[12] For selected reviews on metals-catalyzed formation of C-S, see: (a) Liang, S.; Shaaban, S.; Liu, N.-W.; Hofman, K. Adv. Organomet. Chem. 2018, 69, 135.

For examples on metal-catalyzed formation of $\mathrm{C}-\mathrm{S}$, see: (b) Chen, X.; Hao, X.-S.; Goodhue, C. E.; Yu, J.-Q. J. Am. Chem. Soc. 2006, 128,6790 .

(c) Liu, S.-L.; Li, X.-H.; Zhang, S.-S.; Hou, S.-K.; Yang, G.-C.; Gong, J-F.; Song, M.-P. Adv. Synth. Catal. 2017, 359, 2241.

(d) Liu, C.; Fang, Y.; Wang, S.-Y.; Ji, S.-J. ACS Catal. 2019, 9, 8910.

(e) Wang, X.; Yi, X.; Xu, H.; Dai, H.-X. Org. Lett. 2019, 21, 5981.

(f) Chen, J.; Chen, S.; Xu, X.; Tang, Z.; Au, C.-T.; Qiu, R. J. Org. Chem. 2016, 81, 3246.

(g) Liu, D.; Ma, H.-X.; Fang, P.; Mei, T.-S. Angew. Chem., Int. Ed. 2019, 58, 5033.

(h) Meng, Y.; Wang, M.; Jiang, X. Angew. Chem., Int. Ed. 2020, 59, 134.

[13] Zhao, X.; Dimitrijević, E.; Dong, V. M. J. Am. Chem. Soc. 2009, 131,3466 .

[14] For selected reviews on copper-catalyzed/mediated $\mathrm{C}-\mathrm{H}$ sulfonylation, see: (a) Allen, S. E.; Walvoord, R. R.; Padilla-Salinas, R.; 
Kozlowski, M. C. Chem. Rev. 2013, 113, 6234

(b) Campbell, A. N.; Stahl, S. S. Acc. Chem. Res. 2012, 45, 851.

(c) Zhang, C.; Tang, C.; Jiao, N. Chem. Soc. Rev. 2012, 41, 3464.

(d) Wendlandt, A. E.; Suess, A. M.; Stahl, S. S. Angew. Chem., Int. Ed. 2011, 50, 11062

(e) Daugulis, O.; Do, H.-Q.; Shabashov, D. Acc. Chem. Res. 2009, 42, 1074.

For examples on copper-catalyzed/mediated $\mathrm{C}\left(\mathrm{sp}^{2}\right)-\mathrm{H}$ sulfonylation, see: (g) Uemura, T.; Imoto, S.; Chatani, N. Chem. Lett. 2006, 35,842 .

(h) Peng, J.; Chen, M.; Xie, Z.; Luo, S.; Zhu, Q. Org. Chem. Front. 2014, $1,777$.

(i) Peng, J.; Xie, Z.; Chen, M.; Wang, J.; Zhu, Q. Org. Lett. 2014, 16,4702 .

(j) Hao, X.-Q.; Chen, L.-J.; Ren, B.; Li, L.-Y.; Yang, X.-Y.; Gong, J.-F.; Niu, J.-L.; Song, M.-P. Org. Lett. 2014, 16, 1104.

(k) Guo, Y.; Liu, Z.; Zhu, M.; Li, L.; Li, J.; Zou, D.; Wu, Y.; Wu, Y. Chin. J. Org. Chem. 2020, 40, 724 (in Chinese).

(郭圆圆, 刘振伟, 朱明祥, 李琳琳, 李敬亚, 邹大鹏, 吴豫生, 吴养洁, 有机化学, 2020, 40, 724.)

(1) Fan, C.-L.; Zhang, L.-B.; Liu, J.; Hao, X.-Q.; Niu, J.-L.; Song, M.-P. Org. Chem. Front. 2019, 6, 2215.

For selected reviews on other metals-catalyzed/mediated sulfonylation, see: (m) Liu, J.; Zheng, L. Adv. Synth. Catal. 2019, 361, 1710. (n) Shaaban, S.; Liang, S.; Liu, N.-W.; Manolikakes, G. Org. Biomol. Chem. 2017, 15, 1947.

For examples on other metals-catalyzed/mediated sulfonylation, see: (o) Karmakar, U.; Samanta. R. J. Org. Chem. 2019, 84, 2850.

(p) Kramer, P.; Krieg, S.-C.; Kelm, H. Org. Biomol. Chem. 2019, 17, 5538 .

[15] (a) Liang, S.; Liu, N.-W.; Manolikakes, G. Adv. Synth. Catal. 2016, $358,159$.

(b) Liu, J.; Yu, L.; Zhuang, S.; Gui, Q.; Chen, X.; Wang, W.; Tan, Z. Chem. Commun. 2015, 51, 6418.

(c) Rao, W.-H.; Shi, B.-F. Org. Lett. 2015, 17, 2784.

(d) Liu, S.-L.; Li, X.-H.; Zhang, S.-S.; Hou, S.-K.; Yang, G.-C.; Gong, J.-F.; Song, M.-P. Adv. Synth. Catal. 2017, 359, 2241.

(e) Rao, W.-H.; Zhan, B.-B.; Chen, K.; Ling, P.-X.; Zhang, Z.-Z.; Shi, B.-F. Org. Lett. 2015, 17, 3552.

(f) Wu, Z.; Song, H.; Cui, X.; Pi, C.; Du, W.; Wu, Y. Org. Lett. 2013, 15, 1270.

(g) Yokota, A.; Chatani, N. Chem. Lett. 2015, 44, 902.

(h) Reddy, V. P.; Qiu, R.; Iwasaki, T.; Kambe, N. Org. Biomol. Chem. 2015, 13, 6803. (i) Xia, H.; An, Y.; Zeng, X.; Wu, J. Chem. Commun. 2017, 53, 12548 .

[16] (a) Liang, H.-W.; Jiang, K.; Ding, W.; Yuan, Y.; Shuai, L.; Chen, Y.-C.; Wei, Y. Chem. Commun. 2015, 51, 16928.

(b) Qiao, H.; Sun, S.; Yang, F.; Zhu, Y.; Zhu, W.; Dong, Y.; Wu, Y.; Kong, X.; Jiang, L.; Wu, Y. Org. Lett. 2015, 17, 6086.

(c) Wei, J.; Jiang, J.; Xiao, X.; Lin, D.; Deng, Y.; Ke, Z.; Jiang, H.; Zeng, W. J. Org. Chem. 2016, 81, 946.

(d) Xu, J.; Shen, C.; Zhu, X.; Zhang, P.; Ajitha, M. J.; Huang, K.-W.; An, Z.; Liu, X. Chem.-Asian J. 2016, 11, 882.

(e) Li, J.-M.; Weng, J.; Lu, G.; Chan, A. S. C. Tetrahedron Lett. 2016, 57, 2121.

(f) Liang, S.; Manolikakes, G. Adv. Synth. Catal. 2016, 358, 2371.

(g) Li, J.-M.; Wang, Y.-H.; Yu, Y.; Wu, R.-B.; Weng, J.; Lu, G.. ACS Catal. 2017, 7, 2661

(h) Xia, C.; Wang, K.; Xu, J.; Wei, Z.; Shen, C.; Duan, G.; Zhu, Q.; Zhang, P. RSC Adv. 2016, 6, 37173.

(i) Liang, S.; Bolte, M.; Manolikakes, G. Chem.-Eur. J. 2017, 23, 96.

(j) Bai, P.; Sun, S.; Li, Z.; Qiao, H.; Su, X.; Yang, F.; Wu, Y.; Wu, Y. J. Org. Chem. 2017, 82, 12119.

(k) Chen, G.; Zhang, X.; Zeng, Z.; Peng, W.; Liang, Q.; Liu, J. ChemistrySelect 2017, 2, 1979.

(1) Wang, K.; Wang, G.; Duan, G.; Xia, C. RSC Adv. 2017, 7, 51313. (m) Xia, H.; An, Y.; Zeng, X.; Wu, J. Org. Chem. Front. 2018, 5, 366.

[17] (a) Raziullah.; Kumar, M.; Kant, R.; Koley, D. Adv. Synth. Catal. 2019, 361,1 .

(b) Gandeepan, P.; Koeller, J.; Ackermann, L. ACS Catal. 2017, 7, 1030.

(c) Ahmad, A.; Dutta, H. S.; Khan, B.; Kant, R.; Koley, D. $A d v$. Synth. Catal. 2018, 360, 1644.

(d) Xu, H.; Qiao, X.; Yang, S.; Shen, Z. J. Org. Chem. 2014, 79, 4414.

(e) Guo, X. X.; Gu, D. W.; Wu, Z. X.; Zhang, W. B. Chem. Rev. $\mathbf{2 0 1 5}, 115,1622$

(f) Huffman, L. M.; Stahl, S. S. J. Am. Chem. Soc. 2008, 130, 9196. (g) King, A. E.; Huffman, L. M.; Casitas, A.; Costas, M.; Ribas, X.; Stahl, S. S. J. Am. Chem. Soc. 2010, 132, 12068.

(h) Yao, B.; Wang, Z.-L.; Zhang, H.; Wang, D.-X.; Zhao, L.; Wang, M.-X. J. Org. Chem. 2012, 77, 3336.

(i) Yao, B.; Wang, D.-X.; Huang, Z.-T.; Wang, M.-X. Chem. Commun. 2009, 2899.

[18] Holmes, C. W. N.; Loudon, J. D. J. Chem. Soc. 1940, 1521.

(Zhao, C.) 